\title{
Effect of optical filtering on the performance of 40 Gbps DCDM systems
}

\begin{abstract}
The effect of optical filtering on 40 Gbps on-off keying over 2 x 20, 3 x 13.33, 4 x 10, and 7 x 5.714 Gbps duty-cycle division multiplexing (DCDM) is investigated. The system can tolerate down to $30 \mathrm{GHz}$ optical filter bandwidth for 2 users, and $47.5 \mathrm{GHz}$ for 7 users. At this bandwidth, 40 Gbps was successfully transmitted over $464 \mathrm{~km}$ and $193 \mathrm{~km}$ SSMF using 2 and 7 users, respectively.
\end{abstract}

